\title{
Type 2 Diabetes Mellitus Increases Peritonsillar Abscess Susceptibility: Real-World Evidence
}

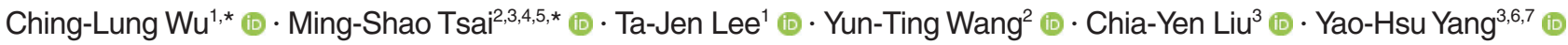 \\ Yao-Te Tsai $^{2,3,5}$ (i) $\cdot$ Cheng-Ming Hsu ${ }^{2,4,5}$ (D) $\cdot$ Ching-Yuan Wu ${ }^{4,6,7}$ (i) $\cdot$ Pey-Jium Chang ${ }^{4}$ (D) $\cdot$ Geng-He Chang ${ }^{2,3,4,5}$ (i) \\ ${ }^{1}$ Department of Otolaryngology-Head and Neck Surgery, Chang Gung Memorial Hospital, Taoyuan; ${ }^{2}$ Department of Otolaryngology-Head and \\ Neck Surgery, Chang Gung Memorial Hospital, Chiayi; ${ }^{3}$ Health Information and Epidemiology Laboratory of Chang Gung Memorial Hospital, \\ Chiayi; ${ }^{4}$ Graduate Institute of Clinical Medical Sciences, College of Medicine, Chang Gung University, Taoyuan; ${ }^{5}$ Faculty of Medicine, College \\ of Medicine, Chang Gung University, Taoyuan; ${ }^{6}$ Department of Traditional Chinese Medicine, Chang Gung Memorial Hospital, Chiayi; \\ ${ }^{7}$ School of Traditional Chinese Medicine, College of Medicine, Chang Gung University, Taoyuan, Taiwan
}

Objectives. Type 2 diabetes mellitus (T2DM) is a risk factor for deep neck infection (DNI) and leads to complications and poor outcomes. Our study aimed to investigate the risk, prognosis, and complications of peritonsillar abscess (PTA) in patients with T2DM.

Methods. We extracted data of patients newly diagnosed as having T2DM between January 2000 and December 2011 from Taiwan's National Health Insurance Research Database. These patients were matched with patients without T2DM, and PTA incidence was compared between both cohorts.

Results. In total, 67,852 patients with and 135,704 patients without T2DM were enrolled. PTA incidence was significantly higher in patients with T2DM (incidence rate ratio, 1.91; $P<0.001$ ); moreover, PTA incidence was higher at 1 to 5 years after T2DM diagnosis than at $<1$ and $>5$ years after T2DM diagnosis. Cox regression analysis showed that patients with T2DM had an approximately 2-fold higher PTA risk (adjusted hazard ratio [aHR]: 1.89, $P<0.001$ ). Patients with a higher adapted Diabetes Complications Severity Index (aDCSI) had higher PTA risk than those with a lower aDCSI (aHRs: 2.17 for aDCSI $\geq 1, P=0.006$ and 1.81 for $\mathrm{aDCSI}=0, P=0.002)$. T2DM patients with a high aDCSI $(\geq 1$ ) had a nonsignificantly longer hospitalization duration and a higher rate of DNI complications than did those with a low aDCSI $(=0)$.

Conclusion. In patients with T2DM, PTA incidence was relatively high, and it increased with T2DM severity. Moreover,T2DM patients should be particularly careful about PTA within 1 to 5 years after the diagnosis, and physicians should keep in mind that the prognosis of PTA was correlated with T2DM severity.

Keywords. Type 2 Diabetes Mellitus; Hyperglycemia; Peritonsillar Abscess; Quinsy; Diabetes Complications Severity Index

\section{INTRODUCTION}

\footnotetext{
- Received November 5, 2020

Revised January 3, 2021

Accepted January 11, 2021

- Corresponding author: Geng-He Chang

Department of Otolaryngology-Head and Neck Surgery, Chiayi Chang

Gung Memorial Hospital, 6,W. Sec., Jiapu Rd, Puzih City,

Chiayi County 613, Taiwan

Tel: +886-975353047, Fax: +886-53623002

E-mail: genghechang@gmail.com

*These authors contributed equally to this work.
}

Peritonsillar abscess (PTA), also termed quinsy, is the accumulation of an abscess between the tonsillar capsule and the superior pharyngeal constrictor muscle [1]. PTA is a common infectious disease estimated to effect 30 of every 100,000 adults yearly in the United States [2]. The pathogenesis of PTA is thought to involve a complication of acute tonsillitis or an infection of minor salivary glands (i.e., Weber's glands) of the superior pole of the tonsils [1]. Patients with PTA are mostly managed using needle aspiration, incision and drainage, or quinsy tonsillectomy in

Copyright (C) 2021 by Korean Society of Otorhinolaryngology-Head and Neck Surgery.

This is an open-access article distributed under the terms of the Creative Commons Attribution Non-Commercial License (https://creativecommons.org/licenses/by-nc/4.0)

which permits unrestricted non-commercial use, distribution, and reproduction in any medium, provided the original work is properly cited. 
combination with antibiotic treatment; generally, the outcomes are favorable [2]. However, without immediate and adequate treatment, PTA can cause life-threatening complications, including an extension of the abscess into deep neck spaces or the mediastinum, airway obstruction, and sepsis [3].

In clinical practice, PTA is thought to develop independently from deep neck infection (DNI) due to the differences in pathogenesis, therapeutic methods, and clinical outcomes. Unlike PTA, the main etiologies of DNI are odontogenic infection or pharyngitis, and transcervical incision and drainage are frequently required treatments [4]. The mortality rate related to DNI has been reported to range from $1 \%$ to $6 \%$ [5]. By contrast, PTA can be generally resolved by adequate treatment; deaths from PTA are rare. Therefore, in most case series analyzing DNI, researchers have excluded PTA and considered PTA as an intraoral abscess rather than a true DNI [6,7].

In DNI patients, comorbid type 2 diabetes mellitus (T2DM) has typically been associated with a higher number of multispace infections, higher complication and tracheostomy rates, longer hospitalization durations, and higher Klebsiella pneumoniae infection prevalence than in patients without T2DM [8]. In addition, nationwide population-based cohort studies [9] have reported a higher DNI risk in patients with T2DM than in those without. However, few studies have discussed the association between PTA and T2DM, and no population-based study has focused on this issue. Therefore, our aim was to comprehensively investigate whether and how T2DM affects PTA incidence, therapy, and prognosis by using data from the National Health Insurance (NHI) Research Database (NHIRD) of Taiwan.

\section{MATERIALS AND METHODS}

\section{Data source}

In 1995, Taiwan's government introduced its compulsory NHI program; by the end of 2018, the NHI had covered almost all Taiwan residents $[10,11]$. In the database, all insurant claims data (e.g., disease diagnoses in clinics, hospitalizations, medication use and dosage, examination, surgery data, and medical expenses) are saved in an electronic format [12]. In NHIRD, patient diagnoses are coded based on the ninth revision (clinical modification) of International Classification of Diseases (ICD-9-CM) [13]. Related medical data and insurer information are delinked

\section{H I G H L I G H T S}

- Patients with diabetes had an approximately two-fold higher risk of developing a peritonsillar abscess (PTA).

- Patients with more severe diabetes had a higher risk of PTA.

- The risk of developing PTA was highest within 1-5 years after the diabetes diagnosis. to provide a database for research use. Therefore, the rights and welfare of the insurants were preserved in this study $[10,11]$. The Institutional Review Board of Chang Gung Medical Foundation approved this study (IRB No. 201900693B1).

Data used in this research were extracted from a representative subset of NHIRD called the Longitudinal Health Insurance Database 2000 (LHID2000) [14], containing medical data of 1 million insurants selected from the 2000 registry of all NHI enrollees randomly through a systematic sampling method. LHID2000 contains all claims data of these enrollees from over 1997 to 2013. According to a National Health Research Institutes report, the differences in the age, sex, or health care costs of the enrollees in LHID2000 and those of all concurrent NHI enrollees are nonsignificant [14].

\section{Study and comparison cohorts}

The study cohort included patients who received T2DM diagnosis during January 2000 to December 2011, whose pertinent data were retrieved from LHID2000. Because patients with T2DM diagnosed before 2000, their diagnostic time may be earlier than 1997, therefore, we cannot clearly define the disease onset for those diabetic patients. In addition, only patients before 2011 were included in order to retain at least a 2-year follow-up period. T2DM diagnosis was confirmed if the records contained $\geq 1$ inpatient claim and $\geq 3$ outpatient claims related to the ICD9-CM code 250.xx. However, patients diagnosed as having type 1 diabetes mellitus (ICD-9-CM codes: 250.01, 250.11, 250.13, $250.21,250.23,250.31,250.33,250.41,250.43,250.51,250.53$, $250.61,250.63,250.71,250.73,250.81,250.83,250.91$, and 250.93) and those with PTA diagnosis before T2DM diagnosis or missing data were excluded (Fig. 1).

For creation of the comparison cohort, patients withoutT2DM diagnosis were recruited from LHID2000 and patients with missing data were excluded. Next, every patient with T2DM diagnosis was matched for sex, urbanization level, age, and income level with two randomly selected patients without T2DM. The urbanization level is the categorization (urban, suburban, and rural areas) of 359 townships in Taiwan according to the following indicators: population density, percentage of residents with college or higher education, percentage of residents over 65 years of age, percentage of agricultural workers and number of physicians per 100,000 people [9].

\section{Main outcome: PTA incidence}

This study mainly tracked and compared PTA incidence in the two groups. We defined PTA occurrence on the basis of the main diagnoses of patients with PTA when they were hospitalized; the relevant ICD-9-CM code is 475. The follow-up duration was from the study's index date to PTA occurrence, death, or December 31, 2013. 


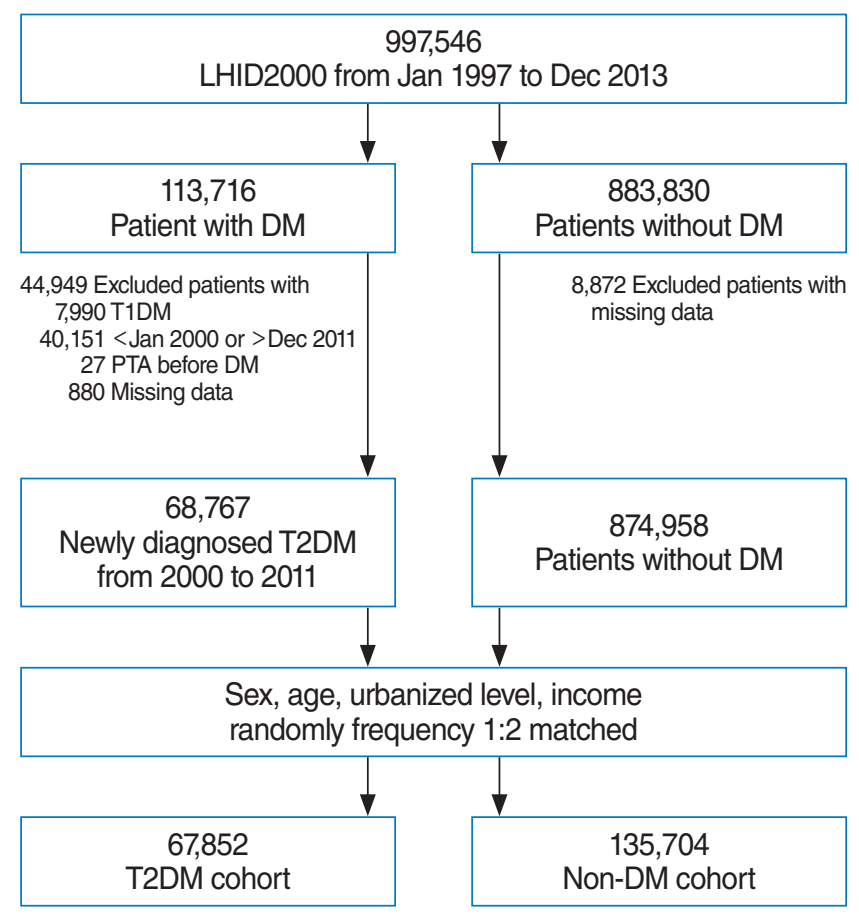

Fig. 1. Flow of cohort enrollment. LHID2000, Longitudinal Health Insurance Database 2000; DM, diabetes mellitus; T1DM, type 1 DM; T2DM, type 2 DM; PTA, peritonsillar abscess.

\section{Comorbidities}

We used ICD-9-CM codes to compare comorbidities between the study and comparison cohorts. Confirmation of a diagnosis required at $\geq 3$ outpatient visits or $\geq 1$ hospitalizations for a particular comorbidity. The included comorbidities (ICD-9-CM codes) were recurrent acute tonsillitis (463), chronic tonsillitis (474.00, 474.01, and 474.02), hypertension (HTN; 401-405), chronic obstructive pulmonary disease (COPD; 491, 492, 494, and 496), liver cirrhosis (LC; 571.2 and 571.5-571.6), chronic kidney disease (CKD; 403, 404, 585, and 586), systemic lupus erythematosus (SLE; 710.0), psoriasis (696.1), Sjögren syndrome (SS; 710.2), smoking related disorders (305.1, 491.2, 492.8, 496, 523.6, and V15.82) and alcohol dependence and abuse (303, 303.xx, 305.0, 305.0x) [13].

\section{T2DM severity}

For evaluating the severity of morbidity related to T2DM, we employed adapted Diabetes Complications Severity Index (aDCSI) [15]. The aDCSI, validated for predicting T2DM-related hospitalization risk [16], can be computed on the basis of ICD-9-CM codes without the use of laboratory data. This index consisted of severity scores from seven complication categories: nephropathy, neuropathy, retinopathy, as well as cardiovascular, metabolic, peripheral vascular, and cerebrovascular diseases. For each category except neuropathy, the score can be 0 (no abnormality), 1 (some abnormality), or 2 (severe abnormality). For neuropathy, the score can be 0 (no abnormality) or 1 (abnormality present).
Total aDCSI can range from 0 to 13 . Here, for further analysis, patients with $\mathrm{T} 2 \mathrm{DM}$ were divided into subgroups using aDCSI= 0 and aDCSI $\geq 1$ (Supplementary Table 1).

\section{Prognosis evaluation}

Hospitalization durations and DNI complication data were collected for prognosis evaluation. The occurrence of a DNI was defined based on the ICD-9-CM codes for Ludwig angina (528.3), parapharyngeal abscess (478.22), retropharyngeal abscess (478.24), or neck cellulitis and abscess (682.1) within the first 3 months after PTA diagnosis.

\section{Statistical analysis}

For comparing the comorbidities and demographic features of our cohorts, we used the unpaired Student $t$-test (for continuous variables) and the Pearson chi-square test (categorical variables). Of these, variables with $P<0.1$ were included in the subsequent multivariate analysis. Next, we applied Kaplan-Meier analysis to determine the cumulative PTA incidence in both cohorts. Differences were ascertained using a log-rank test (two-tailed). Multivariable Cox proportional hazards regression models was employed next determine the hazard ratio (HR) and its 95\% confidence interval (CI) for PTA incidence between our cohorts. We also examined HR stability through sensitivity testing and subgroup analyses as well as elucidated the interaction effect between comorbidities and T2DM on PTA. For all analyses, we utilized SAS ver. 9.4 (SAS Institute, Cary, NC, USA), with significance level set at $P<0.05$.

\section{RESULTS}

After the matching process was completed, the study and comparison cohort included 67,852 and 135,704 patients, respectively. The demographic characteristics of the study and comparison cohorts are summarized in Table 1. In general, patients with T2DM were more likely to have HTN, CKD, COPD, LC, smoking-related disorders, alcohol dependence and abuse, and systemic autoimmune diseases, including psoriasis, SS, and SLE than those without. The mean follow-up duration and PTA incidence rates were, respectively, 7.3 \pm 3.7 years and 12.4 per 100,000 personyears among patients with T2DM and $7.6 \pm 3.6$ years and 6.5 per 100,000 person-years among those without T2DM (Table 2). A significantly higher PTA incidence was observed in patients with $\mathrm{T} 2 \mathrm{DM}$ than in those without (incidence rate ratio [IRR], 1.91; 95\% CI, 1.35-2.70; $P<0.001)$. The average time from T2DM diagnosis to PTA occurrence was $4.2 \pm 3.1$ years. Accordingly, we divided the follow-up periods into $<1,1$ to 5 , and $>5$ years after T2DM diagnosis. A separate analysis of the relative PTA incidence between our two cohorts for the relevant periods revealed that PTA had the highest IRR during 1 to 5 years after T2DM diagnosis (for $<1,1$ to 5 , and $>5$ years after T2DM diagnosis; 
IRR [95\% CI]: 1.72 [0.77-3.83], $P=0.187 ; 2.47$ [1.47-4.16], $P=0.001$; and 1.45 [0.81-2.60], $P=0.209$, respectively).

The subsequent Kaplan-Meier analysis showed that cumula-

Table 1. Demographic and clinical characteristics of the T2DM and non-DM cohorts

\begin{tabular}{|c|c|c|c|}
\hline Characteristic & T2DM & Non-DM & $P$-value \\
\hline Total & 67,852 & 135,704 & \\
\hline Sex & & & $1.000^{\mathrm{a})}$ \\
\hline Male & $36,380(53.6)$ & $72,760(53.6)$ & \\
\hline Female & $31,472(46.4)$ & $62,944(46.4)$ & \\
\hline Age (yr) & & & $1.000^{a)}$ \\
\hline$<60$ & $37,531(55.3)$ & $75,062(55.3)$ & \\
\hline$\geq 60$ & $30,321(44.7)$ & $60,642(44.7)$ & \\
\hline Urbanization level & & & $1.000^{\mathrm{a})}$ \\
\hline 1 (City) & $19,858(29.3)$ & $39,716(29.3)$ & \\
\hline 2 & $30,792(45.4)$ & $61,584(45.4)$ & \\
\hline 3 & $11,329(16.7)$ & $22,658(16.7)$ & \\
\hline 4 (Village) & $5,873(8.7)$ & $11,746(8.7)$ & \\
\hline Income $^{\text {b) }}$ & & & $1.000^{\mathrm{a})}$ \\
\hline 0 & $11,801(17.4)$ & 23,602 (17.4) & \\
\hline $1-15,840$ & $10,582(15.6)$ & $21,164(15.6)$ & \\
\hline $15,841-25,000$ & $31,785(46.8)$ & $63,570(46.8)$ & \\
\hline$\geq 25,001$ & $13,684(20.2)$ & $27,368(20.2)$ & \\
\hline \multicolumn{4}{|l|}{ Comorbidity } \\
\hline Acute tonsillitis & $8,173(12.1)$ & $12,915(9.5)$ & $<0.001^{\text {a) }}$ \\
\hline Chronic tonsillitis & $58(0.1)$ & $107(0.1)$ & 0.620 \\
\hline HTN & $34,647(51.1)$ & $33,479(24.7)$ & $<0.001^{\text {a) }}$ \\
\hline CKD & $2,537(3.7)$ & $2,678(2.0)$ & $<0.001^{\text {a) }}$ \\
\hline COPD & $9,086(13.4)$ & $12,212(9.0)$ & $<0.001^{\text {a) }}$ \\
\hline LC & $1,747(2.6)$ & $1,959(1.4)$ & $<0.001^{\text {a) }}$ \\
\hline Autoimmune $^{\mathrm{C})}$ & $2,073(3.1)$ & $2,913(2.2)$ & $<0.001^{\text {a) }}$ \\
\hline Smoking-related disorder & $5,590(8.24)$ & $7,011(5.17)$ & $<0.001^{\text {a) }}$ \\
\hline $\begin{array}{l}\text { Alcohol dependence and } \\
\text { abuse }\end{array}$ & $616(0.91)$ & $508(0.37)$ & $<0.001^{\text {a) }}$ \\
\hline \multicolumn{4}{|l|}{ Outcome } \\
\hline PTA & $61(0.09)$ & $67(0.05)$ & $<0.001^{\text {a) }}$ \\
\hline
\end{tabular}

Values are presented as number (\%).

T2DM, type 2 diabetes mellitus; HTN, hypertension; CKD, chronic kidney disease; COPD, chronic obstructive pulmonary disease; LC, liver cirrhosis; PTA, peritonsillar abscess.

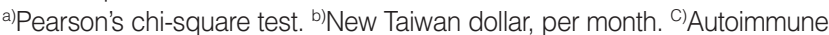
diseases include systemic lupus erythematosus, rheumatoid arthritis, and Sjögren syndrome. tive PTA incidence was significantly higher in patients with T2DM than in those without $(P<0.001)$ (Fig. 2). Next, to approximate the adjusted HRs (aHRs) in both cohorts, multivariable Cox proportional hazards regression was employed (Table 3). In the main model, we adjusted for age, sex, and income and urbanization levels. The full model comprised the main model and all the selected comorbidities (recurrent acute tonsillitis, chronic tonsillitis, HTN, CKD, COPD, LC, autoimmune diseases, smoking-related disorders, and alcohol dependence and abuse). PTA risk was approximately two-fold higher in patients with T2DM than in those without (for the main and full models, aHRs [95\% CIs]: 1.89 [1.34-2.68], $P<0.001$ and 1.71 [1.19-2.46], $P=0.004$, respectively). Of particular note, recurrent acute tonsillitis did not significantly affect the T2DM-associated risk for PTA development.

To determine the constant and independent effects of T2DM among all subgroups, we conducted sensitivity and subgroup analyses. In our sensitivity analysis, through alternately adding one comorbidity to the main model, the PTA risk was noted to be substantial and constant in patients with T2DM. In most of the subgroup analyses, T2DM significantly increased the risk of

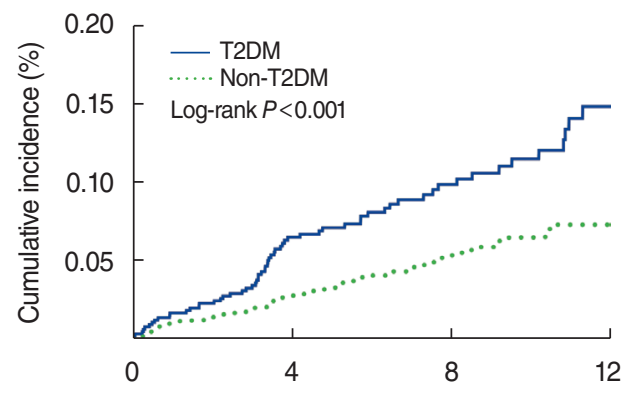

\begin{tabular}{lrrrr}
\hline No. at risk & \multicolumn{4}{c}{ Follow-up time (yr) } \\
\hline T2DM & 67,852 & 51,519 & 28,784 & 9,839 \\
Non-T2DM & 135,704 & 107,723 & 61,603 & 21,565 \\
\hline
\end{tabular}

Fig. 2. Cumulative peritonsillar abscess (PTA) incidence in patients without and with type 2 diabetes mellitus (T2DM). Kaplan-Meier analysis for cumulative PTA incidence in the study and comparison cohorts during the study period (2000-2013). A log-rank test revealed that compared with those without T2DM, patients with T2DM had a significantly higher cumulative PTA incidence $(P<0.001)$.

Table 2. Incidence of PTA in the T2DM and non-DM cohorts

\begin{tabular}{|c|c|c|c|c|c|c|c|c|c|c|}
\hline \multirow{2}{*}{ Variable } & \multicolumn{4}{|c|}{ T2DM } & \multicolumn{4}{|c|}{ Non-DM } & \multirow{2}{*}{ IRR (95\% CI) } & \multirow{2}{*}{$P$-value } \\
\hline & $n$ & PTA & PY & Rate $^{\text {a) }}$ & $n$ & PTA & PY & Rate $^{\text {a) }}$ & & \\
\hline Overall & 67,852 & 61 & 493,627 & 12.4 & 135,704 & 67 & $1,034,104$ & 6.5 & $1.91(1.35-2.70)$ & $<0.001$ \\
\hline \multicolumn{11}{|c|}{ Follow-up (yr) } \\
\hline$<1$ & 67,852 & 11 & 66,678 & 16.5 & 135,704 & 13 & 135,252 & 9.6 & $1.72(0.77-3.83)$ & 0.187 \\
\hline $1-5$ & 65,955 & 31 & 229,895 & 13.5 & 134,772 & 26 & 476,845 & 5.5 & $2.47(1.47-4.16)$ & 0.001 \\
\hline$>5$ & 45,233 & 19 & 197,054 & 9.6 & 95,179 & 28 & 422,007 & 6.6 & $1.45(0.81-2.60)$ & 0.209 \\
\hline
\end{tabular}

PTA, peritonsillar abscess; T2DM, type 2 diabetes mellitus; PY, person-years; IRR, incidence rate ratio; Cl, confidence interval.

a) Rate: per 100,000 person-years; IRR was compared using Poisson regression. 
Table 3. Multivariable Cox proportional hazards regression of the association between T2DM and the risk of PTA

\begin{tabular}{|c|c|c|}
\hline Variable & $\begin{array}{l}\text { Adjusted HR } \\
(95 \% \mathrm{Cl})\end{array}$ & $P$-value \\
\hline \multicolumn{3}{|l|}{ Multivariable regression analysis } \\
\hline Non-DM & Reference & \\
\hline T2DM (main model ${ }^{(a)}$ ) & $1.89(1.34-2.68)$ & $<0.001$ \\
\hline T2DM (full model ${ }^{\mathrm{b})}$ ) & $1.71(1.19-2.46)$ & 0.004 \\
\hline \multicolumn{3}{|l|}{ Sensitivity analysis ${ }^{c)}$} \\
\hline Main model+acute tonsillitis & $1.85(1.31-2.63)$ & 0.001 \\
\hline Main model+chronic tonsillitis & $1.89(1.34-2.68)$ & $<0.001$ \\
\hline Main model+HTN & $1.81(1.26-2.60)$ & 0.001 \\
\hline Main model+CKD & $1.91(1.35-2.70)$ & $<0.001$ \\
\hline Main model+COPD & $1.87(1.32-2.64)$ & $<0.001$ \\
\hline Main model+LC & $1.88(1.33-2.67)$ & $<0.001$ \\
\hline Main model+autoimmune disease & $1.88(1.33-2.66)$ & $<0.001$ \\
\hline Main model+smoking related disorders & $1.89(1.33-2.67)$ & $<0.001$ \\
\hline $\begin{array}{l}\text { Main model+alcohol dependence and } \\
\text { abuse }\end{array}$ & $1.86(1.32-2.64)$ & $<0.001$ \\
\hline \multicolumn{3}{|l|}{ Subgroup analysis ${ }^{\mathrm{d})}$} \\
\hline \multicolumn{3}{|l|}{ Sex } \\
\hline Female & $1.74(0.97-3.10)$ & 0.063 \\
\hline Male & $1.99(1.29-3.07)$ & $<0.001$ \\
\hline \multicolumn{3}{|l|}{ Age } \\
\hline$<60 \mathrm{yr}$ & $2.02(1.35-3.00)$ & 0.001 \\
\hline$\geq 60 \mathrm{yr}$ & $1.53(0.75-3.13)$ & 0.240 \\
\hline \multicolumn{3}{|l|}{ Acute tonsillitis } \\
\hline No & $1.87(1.28-2.74)$ & 0.001 \\
\hline Yes & $1.73(0.73-4.07)$ & 0.211 \\
\hline \multicolumn{3}{|l|}{ Chronic tonsillitis } \\
\hline No & $1.89(1.34-2.68)$ & $<0.001$ \\
\hline Yes & - & - \\
\hline \multicolumn{3}{|l|}{ HTN } \\
\hline No & $1.71(1.11-2.65)$ & 0.016 \\
\hline Yes & $2.09(1.06-4.13)$ & 0.034 \\
\hline \multicolumn{3}{|l|}{ CKD } \\
\hline No & $1.89(1.33-2.67)$ & $<0.001$ \\
\hline Yes & - & - \\
\hline \multicolumn{3}{|l|}{ COPD } \\
\hline No & $1.91(1.32-2.75)$ & 0.001 \\
\hline Yes & $1.57(0.53-4.70)$ & 0.417 \\
\hline \multicolumn{3}{|l|}{ LC } \\
\hline No & $1.88(1.32-2.67)$ & $<0.001$ \\
\hline Yes & $1.78(0.16-19.81)$ & 0.641 \\
\hline \multicolumn{3}{|l|}{ Autoimmune disease } \\
\hline No & $1.96(1.38-2.79)$ & $<0.001$ \\
\hline Yes & $0.36(0.04-3.58)$ & 0.383 \\
\hline
\end{tabular}

T2DM, type 2 diabetes mellitus; PTA, peritonsillar abscess; HR, hazard ratio; $\mathrm{Cl}$, confidence interval; HTN, hypertension; CKD, chronic kidney disease; COPD, chronic obstructive pulmonary disease; LC, liver cirrhosis.

a)Main model was adjusted by sex, age, urbanization, and income level. b)Full model was based on the main model with all comorbidities (acute tonsillitis, chronic tonsillitis, HTN, CKD, LC, COPD, autoimmune diseases, smoking-related disorders, alcohol dependence and abuse). ${ }^{\circ}$ The models were adjusted for covariates in the main model as well as each additional listed comorbidity. ${ }^{d}$ The reference group for the subgroup analysis was the matched non-DM cohort.

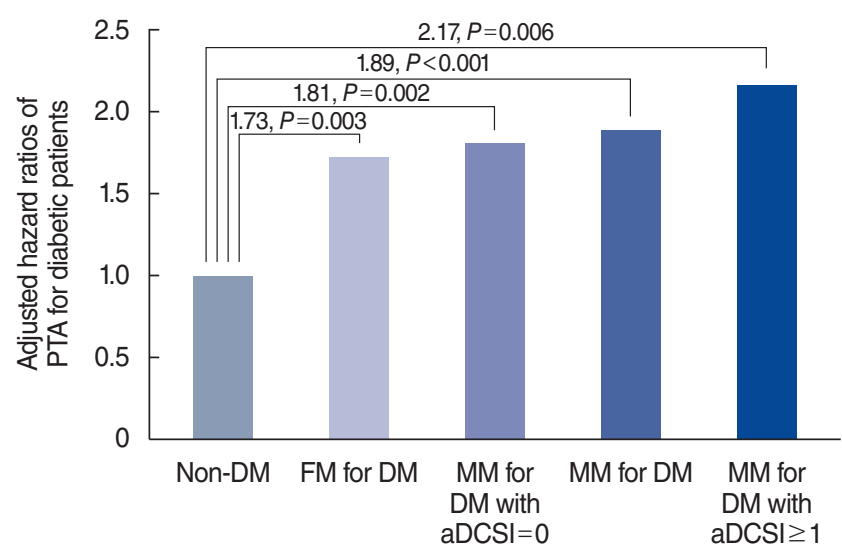

Fig. 3. Peritonsillar abscess (PTA) risk in patients with type 2 diabetes mellitus (T2DM) based on the various Cox regression models. FM, full-model (adding adjustment of all the selected comorbidities, including hypertension, chronic kidney disease, chronic obstructive pulmonary disease, liver cirrhosis, and autoimmune diseases in the main model); MM, main model (Cox regression model with adjustment of age, sex, and income and urbanization levels); aDCSI, adapted Diabetes Complications Severity Index.

PTA; however, in some subgroups, this tendency did not reach statistical significance. Patients with autoimmune diseases showed an insignificant protective effect of PTA. However, the limited case number (less than $3 \%$ of all cases) might have caused a wide confidence interval.

Fig. 3 presents the PTA risk in groups with different levels of T2DM severity. Patients with T2DM were categorized into two subgroups according to their aDCSI (a representative of T2DM severity): those with a lower aDCSI $(=0)$ and those with a higher aDCSI $(\geq 1)$. We evaluated PTA risk in these subgroups using Cox regression models with simultaneous adjustments for age, sex, and urbanization and income levels. The results demonstrated that patients with a higher aDCSI had a higher PTA risk than did those with a lower aDCSI (for the main model with aDCSI $\geq 1$ and $\mathrm{aDCSI}=0$; aHRs [95\% CI]: 2.17 [1.25-3.74], $P=0.006$ and 1.81 [1.24-2.64], $P=0.002$, respectively).

Table 4 presents PTA prognoses in our patients with and without T2DM who developed PTA (T2DM-PTA and non-T2DMPTA groups, respectively). The mean hospitalization duration did not differ significantly between the T2DM-PTA and non-T2DMPTA cohorts, respectively $(9.4 \pm 27.2$ and $5.5 \pm 4.5$ days, respectively; $P=0.271)$. In particular, T2DM-PTA patients with an $\mathrm{aDCSI}=0$ had a mean hospitalization duration $(5.7 \pm 3.6$ days $)$ identical to that of non-T2DM-PTA patients $(P=0.874)$; by contrast, T2DM-PTA patients with an aDCSI $\geq 1$ had a nonsignificantly longer hospitalization duration $(10.7 \pm 31.6$ days) than that of non-T2DM-PTA patients $(P=0.275)$. DNI incidence did not differ significantly between the T2DM-PTA and non-T2DMPTA groups ( $16.4 \%$ and $11.9 \%$, respectively; $P=0.469)$. However,T2DM patients with an aDCSI $\geq 1$ had a higher probability of having PTA and DNI than did their non-T2DM counterparts 
Table 4. Prognoses of the T2DM and non-DM cohorts

\begin{tabular}{|c|c|c|c|c|}
\hline \multirow[b]{2}{*}{ Variable } & \multicolumn{3}{|c|}{ T2DM-PTA } & \multirow{2}{*}{$\begin{array}{c}\text { Non-DM-PTA } \\
\quad(n=67)\end{array}$} \\
\hline & $\begin{array}{l}\text { Total } \\
(n=61)\end{array}$ & $\begin{array}{c}\mathrm{aDCSI} \geq 1 \\
(n=16)\end{array}$ & $\begin{array}{c}\text { aDCSI }=0 \\
(n=45)\end{array}$ & \\
\hline \multicolumn{5}{|l|}{ Severity } \\
\hline Hospitalization (day) & $9.4 \pm 27.2$ & $10.7 \pm 31.6$ & $5.7 \pm 3.6$ & $5.5 \pm 4.5$ \\
\hline$P$-value ${ }^{a)}$ & 0.271 & 0.275 & 0.874 & Reference \\
\hline $\mathrm{DNI}$ & $10(16.4)$ & $9(20.0)$ & $1(6.3)$ & $8(11.9)$ \\
\hline$P$-value ${ }^{\text {b) }}$ & 0.469 & 0.244 & - & Reference \\
\hline Recurrence & $2(3.3)$ & 2 & 0 & $2(3.0)$ \\
\hline$P$-value ${ }^{\text {b) }}$ & 1.000 & 1.000 & - & Reference \\
\hline
\end{tabular}

Values are presented as mean \pm standard deviation or number (\%). T2DM, type 2 diabetes mellitus; PTA, peritonsillar abscess; aDCSI, adapted Diabetes Complications Severity Index; DNI, deep neck infection. a) Student t-test. ${ }^{\text {b) }}$ Fisher exact test.

(DNI in patients with an aDCSI $\geq 1$ vs. non-T2DM-PTA patients: $20.0 \%$ vs. $11.9 \%, P=0.244)$.

Finally, PTA-associated mortality was not observed in either of the two groups; moreover, the proportion of PTA recurrence did not differ between these two groups (as determined at follow-up; PTA recurrence in T2DM vs. non-T2DM cohorts: 3.3\% vs. $3.0 \%, P=1.000)$.

\section{DISCUSSION}

Here, we found that patients with T2DM had a higher PTA incidence than did patients without T2DM; this is the first evidence of this association in the literature.T2DM is a critical health problem worldwide due to its tremendous medical costs and its association with multiorgan complications. In Taiwan, according to the 2017 Statistics on Health Promotion, the prevalence of T2DM or hyperglycemia in the adult population was approximately $10.1 \%$, and T2DM was the fifth leading cause of death in 2017 [17]. Patients who have T2DM are considered immunocompromised due to immune dysfunction and their persistent hyperglycemic status. In patients with T2DM, cellular innate immunity, including complement factors, cytokine response, and monocyte/macrophage function (chemotaxis and phagocytosis), is impaired [18]. Recent studies have revealed T-cell homeostatic dysregulation, dysfunction of natural killer cells and B cells, and abnormal polarization of macrophages in patients who have T2DM; these are related to insulin resistance development and an increased susceptibility to infections [19]. Moreover, T2DM can increase the risks of infections of the lower respiratory tract, urinary tract, skin and mucous membranes, and deep neck space [9]. In the present study, PTA risk was 1.73-fold higher in the T2DM cohort even after adjustment for demographic characteristics and comorbidities. Furthermore, we found that patients with more severe T2DM (aDCSI $\geq 1$ ) had a higher PTA risk than those with less severe T2DM $(\mathrm{aDCSI}=0)$; a similar association with T2DM has also been reported in relation to other infectious diseases [20].

This study revealed that people with T2DM were approximately twice as likely to develop a PTA infection than people without DM. We further subdivided the follow-up duration and found that of the three studied periods, the relative PTA IRR between 1 and 5 years after the diagnosis of T2DM was the highest. In a related study, hemoglobin A1c (HbA1c) levels significantly imrpoved within the first year after a diabetes diagnosis, which indicates that patients with T2DM had better glycemic control in the early stage of the disease [21]. Patient compliance and medication adherence play an important role in glycemic control [22, 23]. We propose that patients had better medication adherence at the early stages after their diagnosis and that their adherence decreased with time. Sustained hyperglycemia or glycemic instability is correlated with several T2DM-related complications [24]. We found that poor glycemic control or glycemic variability after 1 year increased the PTA risk; physicians should pay particular attention to the conditions of patients with glycemic fluctuations because they have a higher susceptibility to infection.

A precise elucidation of PTA incidence is difficult, and it has not been reported in Taiwan thus far. In a national survey of randomly selected physicians in the United States, Herzon and Harris [24] reported that the cumulative PTA incidence was 30.1 per 100,000 person-years. Other reported annual incidence rates of inpatients with PTA were, respectively, 12, 10, and 9 per 100,000 person-years in London (United Kingdom) [25], Northern Ireland [26], and a community in Israel [27]. Only one study was conducted using a national database; Qureshi et al. reported an annual incidence of approximately 5 per 100,000 person-years in adult inpatients in the United States [28]. The differences in reported incidence rates might result from different insurance systems and health care practices. In our study, the incidence of PTA requiring inpatient management was 8.4 per 100,000 person-years, which was within the range reported in the literature. The reliability of the NHIRD and the applicability of its data to real-world settings are therefore reiterated.

According to the relevant literature, under normal circumstances, the time required for patients with PTA to receive treatment for this condition during hospitalization ranges from 2 to 7 days [2]. Furthermore, patients with comorbidities or advanced age require longer hospital stays. In our study, patients with T2DM and PTA had a longer hospitalization duration $(9.4 \pm 27.2$ days $)$ than those reported in previous data, but the length of hospital stay of patients without T2DM was within the general reported duration (5.5 \pm 4.5 days). Although the difference in hospitalization durations was nonsignificant between PTA patients with and without T2DM $(P=0.271)$, PTA patients with T2DM required more time in hospital for treatment. In addition, we further revealed that this longer hospitalization duration was mainly observed for patients with T2DM with more complications or comorbidities (aDCSI $\geq 1$ ). Patients with T2DM without complica- 
tions or comorbidities $(\mathrm{aDCSI}=0)$ received treatment in the hospital for approximately the same duration as patients without T2DM (aDCSI $\geq 1$ vs. $\mathrm{aDCSI}=0$ vs. non-T2DM: $10.7 \pm 31.6$ vs. $5.7 \pm 3.6$ vs. $5.5 \pm 4.5$ days).

In a previous study, the recurrence rate of PTA was approximately $5 \%$, which is close to our result (recurrence rate: $3.3 \%$ and $3.0 \%$ in patients with and without T2DM, respectively). A history of tonsillitis was reported to be a risk factor for PTA recurrence [29]. Additionally, abscess spread to the extraperitonsillar space (based on computed tomography findings) was considered to increase the risk of PTA recurrence [30]. However, no evidence is available to demonstrate that T2DM is associated with PTA recurrence [29,30]. Although our recurrence rate was low, it still aligns with the rates reported in the literature. Evidence in the literature on the correlations of comorbidities such as hypertension, T2DM, and CKD with PTA is limited. The correlation between T2DM and PTA recurrence should be supported by more large-scale population-based research.

Our study has several advantages. First, we included a large number of patients with T2DM in our samples and compared them with a nationally representative control group. As such, our results provide real-world evidence. In addition, this study had a long follow-up period of $>7$ years; thus, we could analyze the time from T2DM diagnosis to the occurrence of PTA, and we could subdivide follow-up durations to analyze PTA incidence during different periods. However, the study had some limitations. First, from this large aforementioned database, we could not obtain records related to clinical symptoms and physical examinations. Second, the database provided no further detailed information, such as records of the treatment process, imaging findings, and laboratory data like $\mathrm{HbA} 1 \mathrm{c}$ or fasting glucose levels; thus, we could assess neither the severity and degree of invasion of PTA nor the diabetes control status of patients. Third, the results of a bacterial culture were not provided in the database. Consequently, we were unable to determine the type of bacteria that caused the infections and analyze results on antibiotic sensitivity. Fourth, this study only included patients with PTA who were hospitalized for treatment, which might have led to an underestimation of the overall number of PTA patients. However, because of the convenience of the health care system in Taiwan, most patients with PTA will be hospitalized for treatment, and recruiting only hospitalized patients could ensure the accuracy of the diagnosis, making the research conscientious and careful. Further investigations related to this missing information would be worthwhile.

In our study, patients with T2DM had a higher PTA risk than did those without T2DM, and more severe T2DM was associated with a higher PTA risk. In addition, patients had the highest PTA risk between 1 and 5 years after T2DM diagnosis. Nevertheless, T2DM itself did not significantly affect the PTA prognosis.

\section{CONFLICT OF INTEREST}

No potential conflict of interest relevant to this article was reported.

\section{ACKNOWLEDGMENTS}

This research was funded by Chiayi Chang Gung Memorial Hospital research projects (grant no. CFRPG6J0081).

We acknowledge the comments and assistance related to data analysis provided by the Health Information and Epidemiology Laboratory, Chiayi Chang Gung Memorial Hospital.

\section{ORCID}

Ching-Lung Wu https://orcid.org/0000-0003-4804-0477

Ming-Shao Tsai https://orcid.org/0000-0002-9314-5940

Ta-Jen Lee https://orcid.org/0000-0003-0508-1359

Yun-Ting Wang https://orcid.org/0000-0001-6190-4351

Chia-Yen Liu https://orcid.org/0000-0002-8350-2684

Yao-Hsu Yang https://orcid.org/0000-0002-8080-0504

Yao-TeTsai https://orcid.org/0000-0002-2313-0890

Cheng-Ming Hsu https://orcid.org/0000-0003-3792-3784

Ching-Yuan Wu https://orcid.org/0000-0001-6172-6647

Pey-Jium Chang https://orcid.org/0000-0002-6492-0346

Geng-He Chang https://orcid.org/0000-0001-5939-9747

\section{AUTHOR CONTRIBUTIONS}

Conceptualization: GHC, MST. Data curation: CYL, YHY. Formal analysis: CYL, YHY. Funding acquisition: GHC. Methodology: GHC, YHY. Project administration: CLW, MST, YTT, CMH. Visualization: CMH, CYW, TJL. Writing-original draft: CLW, MST. Writing-review \& editing: GHC, YTW, PJC, TJL, CMH, YTT, CYW.

\section{SUPPLEMENTARY MATERIALS}

Supplementary materials can be found via https://doi.org/10. 21053/ceo.2020.02257.

\section{REFERENCES}

1. Klug TE, Rusan M, Fuursted K, Ovesen T. Peritonsillar abscess: complication of acute tonsillitis or Weber's glands infection? Otolaryngol Head Neck Surg. 2016 Aug;155(2):199-207.

2. Galioto NJ. Peritonsillar abscess. Am Fam Physician. 2017 Apr;95(8): 
501-6.

3. Powell J, Wilson JA. An evidence-based review of peritonsillar abscess. Clin Otolaryngol. 2012 Apr;37(2):136-45.

4. Chang GH, Ding MC, Chen YC, Yang YH, Liu CY, Chang PJ, et al. Real-world evidence for increased deep neck infection risk in patients with rheumatoid arthritis. Laryngoscope. 2020 Jun;130(6):1402-7.

5. Tsai MS, Chang GH, Chen WM, Liu CY, Lin MH, Chang PJ, et al. The association between decompensated liver cirrhosis and deep neck infection: real-world evidence. Int J Environ Res Public Health. 2019 Oct;16(20):3863.

6. Ridder GJ,Technau-Ihling K, Sander A, Boedeker CC. Spectrum and management of deep neck space infections: an 8-year experience of 234 cases. Otolaryngol Head Neck Surg. 2005 Nov;133(5):709-14.

7. Huang TT, Liu TC, Chen PR, Tseng FY,Yeh TH, Chen YS. Deep neck infection: analysis of 185 cases. Head Neck. 2004 Oct;26(10):85460.

8. Huang TT, Tseng FY, Liu TC, Hsu CJ, Chen YS. Deep neck infection in diabetic patients: comparison of clinical picture and outcomes with nondiabetic patients. Otolaryngol Head Neck Surg. 2005 Jun;132(6): 943-7.

9. Chang GH, Ding MC, Yang YH, Lin YH, Liu CY, Lin MH, et al. High risk of deep neck infection in patients with type 1 diabetes mellitus: a nationwide population-based cohort study. J Clin Med. 2018 Oct; 7(11):385.

10. Chang GH, Chen YC, Lin KM, Yang YH, Liu CY, Lin MH, et al. Realworld database examining the association between Sjogren's syndrome and chronic rhinosinusitis. J Clin Med 2019 Jan;8(2):155.

11. TsaiYT, Huang EI, Chang GH,Tsai MS, Hsu CM, Yang YH, et al. Risk of acute epiglottitis in patients with preexisting diabetes mellitus: a population-based case-control study. PLoS One. 2018 Jun;13(6): e0199036.

12. Chang GH, Su YC, Lin KM, Liu CY, Yang YH, Chang PJ, et al. Deep neck infection in systemic lupus erythematosus patients: real-world evidence. Sci Rep. 2020 Mar;10(1):4133.

13. Chang GH,Tsai MS, Liu CY, Lin MH,TsaiYT, Hsu CM, et al. End-stage renal disease: a risk factor of deep neck infection: a nationwide follow-up study in Taiwan. BMC Infect Dis. 2017 Jun;17(1):424.

14. Tsai MS, Li HY, Huang CG, Wang RY, Chuang LP, Chen NH, et al. Risk of Alzheimer's disease in obstructive sleep apnea patients with or without treatment: real-world evidence. Laryngoscope. 2020 Sep; 130(9):2292-8.

15. Chang HY, Weiner JP, Richards TM, Bleich SN, Segal JB. Validating the adapted Diabetes Complications Severity Index in claims data. Am J Manag Care. 2012 Nov;18(11):721-6.

16. Chen HL, Hsiao FY. Risk of hospitalization and healthcare cost associated with Diabetes Complication Severity Index in Taiwan's Na- tional Health Insurance Research Database. J Diabetes Complications. 2014 Sep-Oct;28(5):612-6.

17. Ministry of Health and Welfare. 2017 Annual report or health promotion administration. Taipei City: Taiwan Ministry of Health and Welfare; 2017.

18. Geerlings SE, Hoepelman AI. Immune dysfunction in patients with diabetes mellitus (DM). FEMS Immunol Med Microbiol. 1999 Dec; 26(3-4):259-65.

19. Zhou T, Hu Z, Yang S, Sun L, Yu Z, Wang G. Role of adaptive and innate immunity in type 2 diabetes mellitus. J Diabetes Res. 2018 Nov; 2018:7457269.

20. Ostergaard L, Mogensen UM, Bundgaard JS, Dahl A, Wang A, TorpPedersen C, et al. Duration and complications of diabetes mellitus and the associated risk of infective endocarditis. Int J Cardiol. 2019 Mar;278:280-4.

21. Ito $\mathrm{H}$, Ishida $\mathrm{H}$, Takeuchi Y,Antoku S, Abe M, Mifune M, et al. Longterm effect of metformin on blood glucose control in non-obese patients with type 2 diabetes mellitus. Nutr Metab (Lond). 2010 Nov; 7:83.

22. Doggrell SA, Warot S. The association between the measurement of adherence to anti-diabetes medicine and the HbA1c. Int J Clin Pharm. 2014 Jun;36(3):488-97.

23. Dandona P. Minimizing glycemic fluctuations in patients with type 2 diabetes: approaches and importance. Diabetes TechnolTher. 2017 Sep;19(9):498-506.

24. Herzon FS. Harris P. Mosher Award thesis. Peritonsillar abscess: incidence, current management practices, and a proposal for treatment guidelines. Laryngoscope. 1995 Aug;105(8 Pt 3 Suppl 74):1-17.

25. Sowerby LJ, Hussain Z, Husein M. The epidemiology, antibiotic resistance and post-discharge course of peritonsillar abscesses in London, Ontario. J Otolaryngol Head Neck Surg. 2013 Jan;42(1):5.

26. Hanna BC, McMullan R, Gallagher G, Hedderwick S. The epidemiology of peritonsillar abscess disease in Northern Ireland. J Infect. 2006 Apr;52(4):247-53.

27. Marom T, Cinamon U, Itskoviz D, Roth Y. Changing trends of peritonsillar abscess. Am J Otolaryngol. 2010 May-Jun;31(3):162-7.

28. Qureshi HA, Ference EH, Tan BK, Chandra RK, Kern RC, Smith SS. National trends in retropharyngeal abscess among adult inpatients with peritonsillar abscess. Otolaryngol Head Neck Surg. 2015 Apr; 152(4):661-6.

29. Wang YP, Wang MC, Lin HC, Chou P. The impact of prior tonsillitis and treatment modality on the recurrence of peritonsillar abscess: a nationwide cohort study. PLoS One. 2014 Oct;9(10):e109887.

30. Chung JH, Lee YC, Shin SY, Eun YG. Risk factors for recurrence of peritonsillar abscess. J Laryngol Otol. 2014 Dec;128(12):1084-8. 